\title{
Black Lives Matter: Warum „Rasse“ nicht aus dem Grundgesetz gestrichen werden darf
}

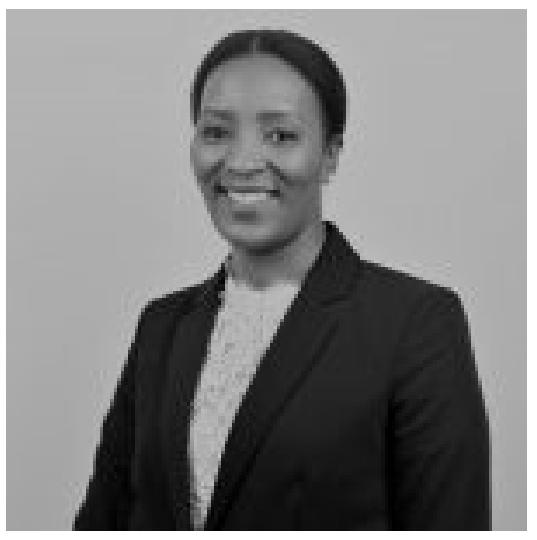

ELISABETH KANEZA

In den USA und auch in Deutschland fordern Schwarze Menschen ihr verfassungsmäßiges Recht ein, nicht durch den Staat diskriminiert, verfolgt und ermordet zu werden. Sie wollen, dass Schwarze Leben in weiß dominierten Gesellschaften zählen. In der öffentlichen Diskussion jedoch scheint daran gezweifelt zu werden, dass Rasse als Rechtsbegriff und Marker von Zugehörigkeit von Bedeutung ist - was sich auch in den Forderungen widerspiegelt, den Begriff aus dem Grundgesetz zu streichen.

Aktuell wird die bereits seit einigen Jahren andauernde Debatte über die Streichung des Begriffs Rasse im Grundgesetz intensiv in den Medien geführt. Erneut angestoßen wurde die Diskussion durch den Mord an dem Afro-Amerikaner George Floyd durch einen weißen Polizisten und die darauffolgenden Proteste der Black Lives Matter Bewegung, die auch hierzulande stattfanden. Die politischen Parteien suchen nun nach Maßnahmen, um den Rassismus in Deutschland zu bekämpfen. Eine dieser Maßnahmen soll die Streichung des Begriffs Rasse aus Art. 3 Abs. 3 S. 1 GG sein. Die Begründung, die vorgebracht wird, ist, dass der Begriff die nationalsozialistische Ideologie von biologischen „Menschenrassen“ propagiere und somit als rassistisch abzulehnen sei.

Vor diesem Hintergrund ist es notwendig, sich mit dem Hintergrund, der Bedeutung und Funktion von Rasse als Rechtsbegriff zu befassen.

\section{Begriffsbestimmung und Entstehungsgeschichte}

Aus Sicht der rechtswissenschaftlichen Critical Race Theory lässt sich

Rasse als eine sozial gewachsene Kategorie definieren, die im Recht als 
Diskriminierungsmerkmal (oder Diskriminierungsgrund) verankert ist, um die Realität von Gruppen anzuerkennen, die als Folge von Zuschreibungsprozessen als rassisch markiert wurden (im Folgenden als Rassialisierung bezeichnet). Rasse dient somit als juristisches Instrument um solche Rassialisierung zu identifizieren und so strukturellen Rassismus zu adressieren. In diesem Artikel wird Rasse kursiv geschrieben, um die Distanz zum vermeintlichen biologischen Gehalt der Terminologie zu kennzeichnen.

Das Verbot der an Rasse anknüpfenden Diskriminierung ist im nationalen Recht, im Unionsrecht sowie im Völkerrecht verankert. Art. 3 Abs. 3 S. 1 GG hat den folgenden Wortlaut:

"Niemand darf wegen seines Geschlechtes, seiner Abstammung, seiner Rasse, seiner Sprache, seiner Heimat und Herkunft, seines Glaubens, seiner religiösen oder politischen Anschauungen benachteiligt werden oder bevorzugt werden."

Die Beratungen des Parlamentarischen Rats, der von 1948 bis 1949 das Grundgesetz erarbeitete, waren von den Eindrücken der NS-Diktatur und des Völkermords an bis zu sechs Millionen europäischen Juden (Holocaust) geprägt. Im Ausschuss für Grundsatzfragen, in dem die Abgeordneten die Formulierungen der Grundrechte entwarfen und vorverhandelten, dienten insbesondere die Verfassungen der Länder sowie der Entwurf der Allgemeinen Erklärung der Menschenrechte (AEMR) der damals noch neuen Organisation der Vereinten Nationen (VN) als Vorlagen für Art. 3 Abs. 3 S.1 GG. In Art. 2 AEMR werden die Menschenrechte ohne Unterschied nach Rasse garantiert.

In Anerkennung des nationalsozialistischen Unrechts wurde mit Art. 116 GG garantiert, dass frühere deutsche Staatsangehörige, denen die Staatsbürgerschaft aus „rassischen Gründen“ entzogen worden ist, wiedereingebürgert werden können. Ferner gab das Bundesentschädigungsgesetz von 1956 Personen, die „aus Gründen der Rasse“ ( 1 Abs. 1) verfolgt worden waren, einen Anspruch auf Entschädigung. Strafrechtlich schützt $\S 130$ StGB „nationale, rassische, religiöse oder durch ethnische Herkunft bestimmte Gruppen" vor der Volksverhetzung. Seit 2006 ist Rasse im Privatrecht durch das Allgemeine Gleichbehandlungsgesetz als Diskriminierungsgrund aufgeführt.

Auf der Ebene des Völkerrechts wurde der erste rechtlich bindende universelle Menschenrechtsvertrag, das Internationale Übereinkommen zur Beseitigung jeder Form der Rassendiskriminierung (International Convention on the Elimination of All Forms of Racial Discrimination, ICERD), 1965 beschlossen. Zu den Entwicklungen, die den Anlass für die ICERD gaben, zählen der Widerstand gegen das Apartheidregime in Südafrika sowie die Bürgerrechtsbewegung der Schwarzen Bevölkerung in den USA. Art. 1 ICERD erkennt Rasse, Hautfarbe, Abstammung, nationalen Ursprung und Volkstum als Diskriminierungsmerkmale an. Neben den Menschenrechtsverträgen der VN ist Rasse in Art. 14 der Europäischen Menschenrechtskonvention sowie in Art. 21 der Grundrechtecharta der Europäischen Union als Diskriminierungsmerkmal geschützt. 
Die historische Betrachtung verdeutlicht, dass es nicht Zweck der Aufnahme des Begriffs Rasse im nationalen Recht, Unionsrecht und Völkerrecht war, die Rassenlehre des Nationalsozialismus, des Kolonialismus und der anti-Schwarzen Segregation zu reproduzieren, sondern dass damit jegliche Diskriminierung auf Grund der Vorstellung von Rasse verboten und Gleichberechtigung ermöglicht werden sollte. Rasse als Diskriminierungsmerkmal zu bestimmen, war die juristische Antwort auf die Realität des anhaltenden strukturellen Rassismus. Als Rechtsbegriff kommt daher Rasse keine biologische Deutung zu.

\section{Funktion von Rasse im Recht: Anerkennung und Abwehrrecht}

Rasse drückt also nicht aus, dass es biologische „Menschenrassen“ gibt, sondern, dass es Personen und Gruppen gibt, die aufgrund dieses Merkmals Ungleichbehandlung erfahren.

Die erste Funktion von Rasse im Recht ist somit, anzuerkennen, dass sowohl rassialisierte Gruppen als auch auf Rassismus basierende Hierarchien existieren und dass die daraus resultierenden Strukturen eine Gleichstellung verhindern. Das im Grundgesetz verankerte Gleichheitsgebot trägt dem Rechnung. Auch in den menschenrechtlichen Verträgen werden die betroffenen Gruppen sowie die Notwendigkeit für die Förderung ihrer Rechte anerkannt. So verpflichtet Art. 2 Abs. 2 ICERD die Vertragsstaaten „auf sozialem, wirtschaftlichem, kulturellem und sonstigem Gebiet besondere und konkrete Maßnahmen, um die Entwicklung und einen hinreichenden Schutz bestimmter Rassengruppen oder ihnen angehörender Einzelpersonen sicher zu stellen, damit gewährleistet wird, dass sie in vollem Umfang und gleichberechtigt in den Genuss der Menschenrechte und Grundfreiheiten gelangen“ zu treffen. Der VN-Menschenrechtsausschuss CERD vertritt die Position, dass die Aufnahme von Rasse in den Verfassungen der Vertragsstaaten notwendig ist, um den Diskriminierungsschutz des ICERD für betroffene Gruppen zu garantieren. In seinen Abschließenden Bemerkungen zum letzten periodischen Staatenbericht Deutschlands (2015) forderte der Ausschuss die Bundesregierung auf, eine Diskriminierungsdefinition im Grundgesetz zu verwenden, die mit Art. 1 ICERD vollständig übereinstimmt.

Neben dieser rechtlichen Relevanz ist Rasse auch von politischer Bedeutung, vor allem dann, wenn durch gezielte Maßnahmen die Situation von betroffenen Gruppen erfasst und verbessert werden soll. Denn rechtlicher Schutz vor Diskriminierung setzt voraus, dass diese erfassbar ist. Ob sie rechtlich erfassbar ist, hängt wiederum davon ab, ob das Gesetz das zutreffende Diskriminierungsmerkmal anerkennt. So schuf die Anerkennung von Rasse eine rechtliche Grundlage für die Wiedergutmachungspolitik der Bundesrepublik, die seit der Nachkriegszeit den Holocaustüberlenden zugute kommt. Bis zu 1 Milliarde Euro gibt die Bundesregierung jährlich für Leistungen der öffentlichen Hand aus.

Die zweite Funktion des Merkmals Rasse im öffentlichen Recht ist das Abwehrrecht gegen den Staat. Das Diskriminierungsverbot des Grundgesetzes richtet sich in erster Linie an die Träger*innen der staatlichen Gewalt - sie sind die Grundrechtsverpflichteten. Gemäß Art. 3 Abs. 3 S.1 GG darf der Staat nicht 
aufgrund des Merkmals der Rasse diskriminieren - aus diesem Grund sind Racial Profiling und Polizeigewalt, die an der Hautfarbe anknüpft sowohl als verfassungs- als auch menschenrechtswidrig zu bewerten. Nach dem in der ICERD verankerten Diskriminierungsverbot haben Staaten „Handlungen oder Praktiken der Rassendiskriminierung gegenüber Personen, Personengruppen oder Einrichtungen zu unterlassen und dafür zu sorgen, dass alle staatlichen und örtlichen Behörden und öffentlichen Einrichtungen im Einklang mit dieser Verpflichtung handeln“ (Art. 2 Abs. 1a).

Somit lässt sich sowohl aus dem nationalen Recht als auch aus dem Völkerrecht ein Verbot der rassischen Diskriminierung ableiten. Personen und Gruppen dürfen nicht aufgrund ihrer Zugehörigkeit zu einer vermeintlichen Rasse benachteiligt werden. Es geht also nicht bloß um die Sanktionierung individualisierten Verhaltens, das als rassistisch gedeutet werden kann, sondern um die Vermeidung und Beseitigung von struktureller Benachteiligung einzelner Gruppen vs. die Bevorzugung anderer Gruppen. Bei der rassischen Diskriminierung ist es daher ausschlaggebend, ob eine Handlung oder Praxis auf das Merkmal der Rasse zweckgerichtet ist und ob für eine oder mehrere Personengruppen dadurch eine Benachteiligung entsteht (i.S. einer Merkmalsträgerschaft). Gemäß der Rechtsprechung des Gerichtshofs der Europäischen Union trifft eine unmittelbare rassische Diskriminierung von Personen und Gruppen auch dann zu, wenn es kein identifizierbares Opfer gibt.

Aufgrund des weit verbreiteten Unbehagens in Deutschland gegenüber der Verwendung des Begriffs Rasse, wird im politischen und gesellschaftlichen Diskurs stattdessen von „rassistisch“ gesprochen. Auf das Recht übertragen, bedeutet es jedoch, dass damit Sinn und Zweck der entsprechenden Rechtsnormen verändert würden. Bereits jetzt weist die deutsche Rechtspraxis keinen Konsens darüber auf, wann staatliches Handeln als rassistisch gilt. So wird beispielsweise die Frage, ob die Praxis des Racial Profiling rassistisch sei, von Richter*innen und Behörden unterschiedlich bewertet. Es ist zudem ein „farbenblinder" Ansatz in der Rassismusbekämpfung zu beobachten, der sich beispielsweise dadurch ausdrückt, dass bei den Straftaten, die als politisch motiviert bestimmt werden, die Hautfarbe der Opfer nicht erfasst wird. Die Bundesregierung räumte in ihrem letzten Staatenbericht an CERD (2013) ein, dass sie keine Angaben über die Zahl rassisch-motivierter Straftaten gegen Schwarze Menschen machen könne, da sie für diese Gruppe nicht separat erfasst werden. Damit ergibt sich ein folgenschweres Problem: Rassische Gewalt an Schwarzen Menschen existiert, statistisch gesehen, nicht. Um diese strukturelle Unsichtbarkeit der Rassismuserfahrungen von Schwarzen Menschen zu adressieren, muss Rasse im Recht benannt und in der strafrechtlichen Praxis erfasst werden.

\section{Umgang mit Rasse im Grundgesetz}

Rasse aus dem Grundgesetz zu streichen würde ein Weniger an Diskriminierungsschutz und Anerkennung für Personen und Gruppen bedeuten, die von Rassismus betroffen sind. Denn fällt ein Merkmal im Antidiskriminierungsrecht weg, besteht die Gefahr, dass für die vormals hiervon erfasste Gruppe eine Schutzlücke entsteht. Ferner würde im nationalen Recht ein dogmatischer 
Widerspruch entstehen, da Rasse als Merkmal in anderen Rechtsnormen wie oben aufgeführt verankert ist.

Politische Parteien sollten jetzt nicht aktionistisch vorpreschen, sondern fachliche Beratungen von Wissenschaftler*innen, insbesondere Forscher*innen of Color, einholen, wie mit dem Merkmal Rasse im Grundgesetz umzugehen ist. Bundestag und Bundesregierung müssen vor allem die völkerrechtlichen Folgen berücksichtigen, die daraus resultieren können, dass Rasse in der Verfassung der Bundesrepublik „entnannt“ wird. Als Vertragsstaat von ICERD ist Deutschland verpflichtet, die effektive Umsetzung des Übereinkommens sicherzustellen. Diese Verpflichtung besteht auch für andere menschenrechtliche Verträge sowie für die Verträge und entsprechenden Richtlinien der Europäischen Union, in denen Rasse als Diskriminierungsmerkmal geführt ist.

Die Befürworter*innen der Streichung verkennen, dass Rassismus und die Vorstellung von Rasse nicht voneinander losgelöst werden können. Es sind zwei Seiten einer Medaille. Die bereits seit Jahrhunderten andauernden Zuschreibungsprozesse sowie die Erfahrungen von Segregation und Gewalt haben Identitäten und Denkmuster hervorgebracht, die rassialisierte Ungleichheit in der Rechtswirklichkeit zur Folge hatten und haben. Die Black Lives Matter Bewegung drückt aus, dass diese Realität kein Relikt der Vergangenheit ist, sondern, dass sie auch in unserer Gegenwart existiert und nicht mehr geleugnet werden kann. Rasse als juristisches Instrument, das diese Ungleichheit benennt und sichtbar macht, zu „entsorgen“, ist keine Handlung gegen den Rassismus. Deshalb gilt: Damit Schwarze Leben und die anderer rassisch markierter Gruppen in Deutschland zählen können, muss Rasse als geschütztes Diskriminierungsmerkmal im Grundgesetz verankert bleiben.

(cc) $\mathrm{BY}-\mathrm{Ne}-\mathrm{ND}$ 